\title{
Social Ties and Mental Health
}

\author{
Ichiro Kawachi and Lisa F. Berkman
}

\begin{abstract}
It is generally agreed that social ties play a beneficial role in the maintenance of psychological well-being. In this targeted review, we highlight four sets of insights that emerge from the literature on social ties and mental health outcomes (defined as stress reactions, psychological well-being, and psychological distress, including depressive symptoms and anxiety). First, the pathways by which social networks and social supports influence mental health can be described by two alternative (although not mutually exclusive) causal models-the main effect model and the stress-buffering model. Second, the protective effects of social ties on mental health are not uniform across groups in society. Gender differences in support derived from social network participation may partly account for the higher prevalence of psychological distress among women compared to men. Social connections may paradoxically increase levels of mental illness symptoms among women with low resources, especially if such connections entail role strain associated with obligations to provide social support to others. Third, egocentric networks are nested within a broader structure of social relationships. The notion of social capital embraces the embeddedness of individual social ties within the broader social structure. Fourth, despite some successes reported in social support interventions to enhance mental bealth, further work is needed to deepen our understanding of the design, timing, and dose of interventions that work, as well as the characteristics of individuals who benefit the most.
\end{abstract}

KEYWORDS Mental health, Psychological distress, Social capital, Social integration, Social ties.

\section{INTRODUCTION}

The link between social isolation and reduced psychological well-being is well established in sociology, dating back to Durkheim. ${ }^{1}$ Smaller social networks, fewer close relationships, and lower perceived adequacy of social support have all been linked to depressive symptoms. ${ }^{2}$ However, the challenges of establishing a causal relationship to social ties are generally greater for mental health than they are for other health outcomes. An obvious threat to validity in cross-sectional studies is the potential bias in the retrospective recall of social ties among distressed individuals. Even with a longitudinal study design, it can be extraordinarily difficult to distinguish between lack of social ties as an antecedent to, or as a concomitant of, psychological distress. For example, it is plausible that certain personality traits, such as introversion, are associated with both lack of social network participation and the occurrence of depressive symptoms. ${ }^{2}$ The difficulties are compounded by

Drs. Kawachi and Berkman are with the Department of Health and Social Behavior, Harvard Center for Society and Health, Harvard School of Public Health, Boston, Massachusetts.

Correspondence: Dr. I. Kawachi, MD, PhD, Department of Health and Social Behavior, Harvard School of Public Health, 677 Huntington Avenue, Boston, MA 02115. (E-mail: Ichiro.Kawachi@ channing.harvard.edu) 
the sparseness of studies that sampled a genuinely premorbid group of individuals. Since lack of social ties can be a consequence of depressive symptoms, the distribution of social ties in a population may reflect reverse causation at baseline (symptoms $\geq$ social isolation), even in a longitudinal follow-up sample.

Such difficulties notwithstanding, most researchers now agree that social ties have a salutary effect on mental health and psychological well-being. ${ }^{2}$ The purpose of this article is not to review the existing studies of social ties and mental health (which are numerous). Nor do we aim to cover the relationships between social ties and specific mental disorders or the role of urbanization/modernization in relation to social ties. Instead, our purpose is to highlight four distinct issues that emerge from the literature on social ties and mental health outcomes, hereby defined as stress reactions, psychological well-being, and symptoms of psychological distress, including depressive symptoms and anxiety. First, we provide a summary of what we know about the mechanisms underlying the link between social ties and mental health, drawing on the conceptual frameworks advanced by Cohen and Wills. ${ }^{3}$ Second, we describe the effect modification of the relationship between social ties and mental health by gender, socioeconomic position, and stage of the life. Third, we describe the relevance of the concept of social capital in this field, drawing on recent theoretical developments in sociology. Last, we briefly describe the state of knowledge about social support interventions to improve mental health.

\section{MECHANISMS UNDERLYING THE RELATION BETWEEN SOCIAL TIES AND MENTAL HEALTH}

Two models have been put forward by Cohen and Wills ${ }^{3}$ to explain the mechanisms by which social relationships influence health outcomes: main effect model and the stress-buffering model. The stress-buffering model posits that social ties are related to well-being only for persons under stress, whereas the main effects model proposes that social relationships have a beneficial effect regardless of whether individuals are under stress. The two models are not mutually exclusive; rather, they may help to explain the influence of specific aspects of social relationships on psychological health. It has been suggested that structural aspects of social relationships (e.g., social networks, social integration) may operate via main effects, whereas functional aspects of social relationships (e.g., perceived support) operate through a stress-buffering mechanism. For instance, the perceived availability of functional support is thought to buffer the effects of stress by enhancing an individual's coping abilities. In contrast, the degree of integration in a social network is postulated to have a direct effect on well-being regardless of the presence of stressful circumstances. ${ }^{4}$

The main effect model (Fig. 1) describes several pathways through which participation in social networks can affect psychological well-being. Social influence refers to the way members of a social network obtain normative guidance about health-relevant behaviors, such as physical activity or cigarette smoking. ${ }^{4}$ Behaviors such as regular exercise may, in turn, exert a salutary influence on mental health. (We should not forget, of course, that norms within some networks might actually encourage smoking and sedentary behavior!) Integration in a social network may also directly produce positive psychological states, including a sense of purpose, belonging, and security, as well as recognition of self-worth. ${ }^{5}$ These positive psychological states, in turn, may benefit mental health because of increased motivation for self-care (e.g., regular exercise, moderation of alcohol intake), as well as the 


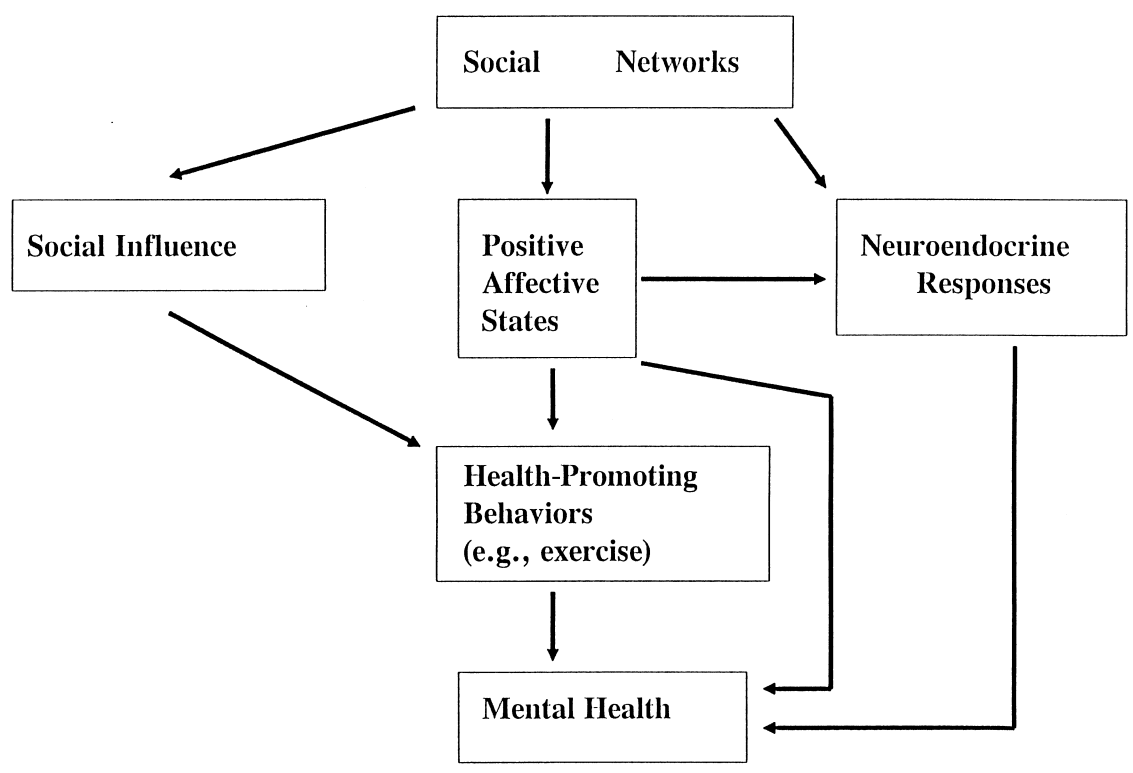

FIGURE 1. Main effect model of social ties and mental health. Adapted from Cohen S, Underwood LG, Gottlieb BH. Social Support Measurement and Intervention. A Guide for Health and Social Scientists. New York: Oxford University Press; 2000.

modulation of the neuroendocrine response to stress. Finally, location in the broader social structure (e.g., participation in community organizations, involvement in social networks, and immersion in intimate relationships) enhances the likelihood of accessing various forms of support, which in turn protect against distress. ${ }^{6}$ Examples of such resources include access to health-relevant information or receipt of informal health care that could prevent a minor ailment from progressing into a more serious psychiatric disorder. ${ }^{5}$ It is also important to recognize that many life events traditionally conceptualized are actually breaks in social ties (e.g., divorce, deaths of loved ones, etc.). Other times, social networks may influence the odds of experiencing a life event (e.g., unemployment).

In the stress-buffering model (Fig. 2), social support is hypothesized to prevent or modulate responses to stressful events that are damaging to health. Support may thus act on several different points in the pathway between stressful events and eventual mental illness. First, the perceived availability of social support in the face of a stressful event may lead to a more benign appraisal of the situation, thereby preventing a cascade of ensuing negative emotional and behavioral responses. ${ }^{7}$ Further downstream, perceived or received support may either reduce the negative emotional reaction to a stressful event or dampen the physiologic/behavioral responses to stress. For instance, in studies of cardiovascular reactivity in which subjects are given a challenge related to public speaking, the availability of support (not actually provided) dampens cardiovascular reactivity. ${ }^{8}$

\section{MODIFIERS OF THE RELATIONSHIP BETWEEN SOCIAL TIES AND MENTAL HEALTH}

The costs and benefits of social ties are not randomly distributed in the population, but vary systematically with gender, socioeconomic position, and stage in life. Cer- 


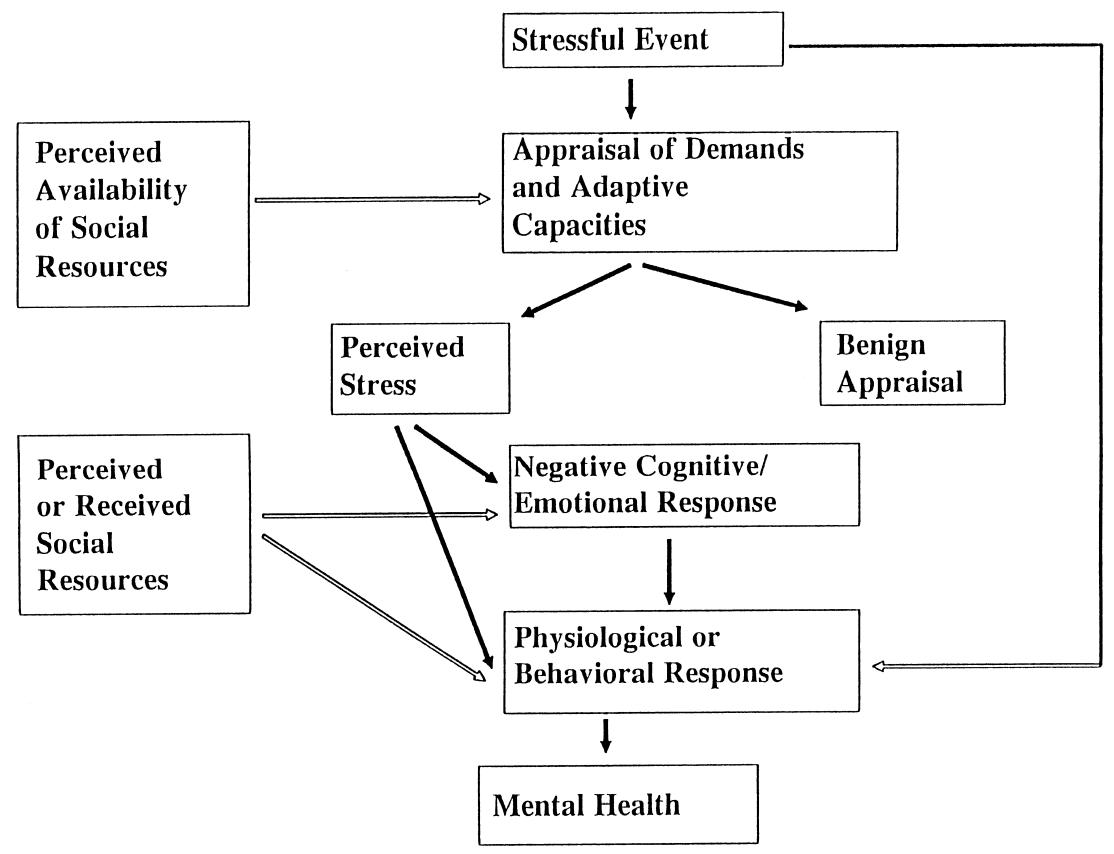

FIGURE 2. Stress-buffering model of social ties and mental health. Adapted from Cohen S, Underwood LG, Gottlieb BH. Social Support Measurement and Intervention. A Guide for Health and Social Scientists. New York: Oxford University Press; 2000.

tain stages in the life course are clearly critical in terms of social relationships. Thus, emotional support during childhood from parents or caregivers has been shown to influence the risk of subsequent depression. ${ }^{9}$ Attachment in early life is critical to psychological development. ${ }^{10,11}$ At the opposite end of the life course, social isolation and loss of social ties are among the most potent predictors of depressive symptoms among the elderly. ${ }^{12}$ On the other hand, social support received from children can paradoxically reinforce a sense of dependence in the elderly, thereby undermining self-esteem and leading to feelings of helplessness. ${ }^{13}$ We suspect that social support can either promote a sense of self-efficacy and self-esteem or become "dis-abling" by reinforcing dependence; therefore, social support can have "mixed" effects. ${ }^{4,14}$

An important new area of research relates the impact of social networks and support to cognitive function among the elderly. Over the last 5 years, three studies have shown that social participation, ${ }^{15}$ social engagement, ${ }^{16}$ or social networks ${ }^{17}$ have predicted dementia or cognitive decline in men and women over the age of 65 years. All of these studies were longitudinal and controlled for baseline cognitive function. While this does not preclude that social withdrawal is in some way a prodromal symptom or marker of early decline, the strength of the study designs and the magnitude of effect size are intriguing.

The effects of social ties on mental health differ also by gender. It has been widely documented that women report significantly higher rates of psychological distress than men, a finding that may be partly explained by gender differences in social network involvement. ${ }^{18}$ Summarizing these gender differences, Belle ${ }^{19}$ observed that women tend to (1) maintain more emotionally intimate relationships 
than men, (2) mobilize more social supports during periods of stress than men, and (3) provide more frequent and more effective social support to others than do men.

With regard to the first observation, women's propensity for intimate social involvements may predispose them to the "contagion of stress" when stressful life events afflict those to whom they feel emotionally close. In other words, women suffer more from other people's problems. Indeed, studies that have separated the stressful components of social networks from the supportive components have found that the former are more strongly related to women's mental health status than the latter. ${ }^{20}$

With regard to the second observation that women tend to mobilize more support than men during periods of stress, research has established that widowhood is consistently more damaging to the mental and physical health of men than of women. ${ }^{21}$ This finding has been attributed to the fact that men's mobilization of support is heavily focused on their spouses, whereas women are much more likely to rely on a child, close relative, or a friend as their confidant. ${ }^{22}$ Interestingly, among couples, supportive aspects of the marital relationship (e.g., satisfaction with spouse, spouse as confidant) appear to be much more strongly linked to the mental health of women than of men. ${ }^{2}$ This may reflect the fact that gender inequalities in domestic relationships-for example, household authority and bargaining power with respect to access to financial resources-result in women being more exposed to the vagaries of their partners' support.

Third, the finding that women tend to provide more frequent social support than men can result in what Belle ${ }^{19}$ termed the "support gap." In dyadic relationships, when the flow of support is highly unequal between women and men, the result may be demoralization and depression.

In addition to these gender differences, the effects of social networks and support on mental health also vary by socioeconomic position. For instance, participation in social networks may be more harmful than helpful for women with low resources, who often face greater difficulty in responding to the needs of network members. ${ }^{19}$ Indeed, it has been noted that those most in need of support from their social networks are often those least likely to receive the support, while they also disproportionately bear the costs of network involvement. ${ }^{23}$

From the recipient's perspective, social support may also entail psychological costs in the sense of indebtedness and obligation. This sense of obligation may itself be patterned by gender. For example, in a study of a southern black community, Dressler and Badger ${ }^{24}$ found that social support from extended family was beneficial for the mental health of men, but not of women. Among women in this community who were 17 to 35 years old, more support from extended kin was associated with more mental health symptoms, a finding that the author attributed to the heavy obligations incurred by young women who received such support. If they received support, women in this community were expected to follow closely the advice of older kin, resulting in high psychological costs associated with conformity. ${ }^{25}$

\section{CONTEXTUAL DIMENSION OF SOCIAL TIES}

To this point, we have discussed social ties from an egocentric perspective. The bulk of epidemiologic investigations of social ties and health has approached the measurement of social ties from an egocentric perspective. That is, investigators 
have typically asked individual subjects about the structure and function of networks that immediately surround them. But, social networks and social support are embedded within a broader set of macrosocial exchanges. As Lin and colleagues, ${ }^{6}$ Boissevain, ${ }^{26}$ and Berkman ${ }^{27}$ note, human relations consist of multiple layers that extending out from the ego. These layers extend from the most intimate relations (e.g., marital ties), outward to social networks (e.g., connections to close relatives and friends), and to "weak" ties consisting of involvement in community, voluntary, and religious organizations. Participation in the last set of ties does not necessarily impose intense person-to-person interactions. Nonetheless, it provides a sense of belongingness and general social identity, which sociological theorists have argued as being relevant for the promotion of psychological well-being. ${ }^{1,28}$

The three layers of social ties have been characterized by Lin et al. ${ }^{6}$ as the "belongingness-bonding-binding" continuum. The notion of an outer zone of connectedness to the wider community resonates with the emerging concept of social capital. ${ }^{29,30}$ Theoretically, all three zones of social ties may exert an independent influence on mental health outcomes. Moreover, Lin et al. ${ }^{6}$ hypothesize that each inner layer is contingent on the outer layers. That is, each outer layer of linkages affords the opportunity to establish inner-layer linkages. For example, belongingness promotes bonding (e.g., community participation increases the likelihood of constructing and maintaining interactive ties in social networks). Some empirical tests bear out this nested structure of social relations, as well as the independent influence of each level on depressive symptoms. ${ }^{6}$

The notion that an individual's egocentric ties are contingent on structural characteristics (e.g., the density of civic associations or the extent of voluntarism in a community) is one of the key insights of social capital theory. ${ }^{30}$ Sociometric analyses are ideal for assessing the collective dimension of social ties ("network of networks"), but they have seldom been employed in epidemiologic investigations. An important task for empirical investigation is therefore to demonstrate the contextual influence of social capital (e.g., in the form of community and workplace social structures) on individual networks and support and on individual-level mental health outcomes.

At the same time, caution must be exercised in assuming that tight-knit social structures always lead to better mental health outcomes. In fact, quite the opposite may be true. The "dark side" of social capital for mental health is illustrated by the classic studies of Brown and Harris ${ }^{31}$ comparing the prevalence of mental illness among women in two areas of Outer Hebrides. ${ }^{31}$ In these rural, Gaelic-speaking communities, a two-factor index of women's participation in traditional life (regular church-going and craft work) predicted lower rates of depression, but also a much higher rate of anxiety disorders. In other words, adherence to traditional forms of social engagement appeared to have simultaneously protective and damaging effects. To explain this discrepancy, the authors referred to Durkheim's distinction between two aspects of traditional societies: réglementation (social regulation) and integration (social cohesiveness). They hypothesized that, while cohesiveness protects against depressive illness, the repressive nature of social regulation was associated with an excess of anxiety disorders. (Similar findings have been noted with regard to the experience of widowhood. ${ }^{32}$ ) Similarly oppressive consequences might be imagined for the mental health of individuals who reside in close-knit communities, who do not happen to "conform" (e.g., because of sexual orientation or the assertion of feminist beliefs or because they are a small minority group in a tightly knit culture of the majority). 


\section{SOCIAL SUPPORT INTERVENTIONS TO IMPROVE MENTAL HEALTH}

The conundrums of establishing the causal effects of social ties on mental health (discussed at the beginning of this review) are best settled by randomized interventions. Yet, despite nearly two decades of interventions to manipulate and enhance social support for health improvement, a recent survey of the field concluded that "there is more evidence of the feasibility of marshaling support than of its effectiveness." ${ }^{\text {(p } 16)}$ There is an urgent need to uncover how interventions that work to strengthen existing network structures and support compare with interventions aimed at creating new communities and networks.

Some successes have been described in the intervention literature. These interventions have taken the form of support group interventions, ${ }^{33}$ one-to-one support interventions, ${ }^{34,35}$ or interventions to enhance natural networks. ${ }^{36}$ Mittleman and colleagues $^{33}$ applied a multifaceted intervention to reduce depressive symptoms among 206 spouse caregivers of patients with Alzheimer's disease. The intervention included six sessions of individual and family counseling, followed by attendance at a support group that met for an indefinite period of time. The counseling sessions targeted communication between family members and taught techniques of problem solving for the caregiver. Caregivers in the intervention group were less depressed than those in the control group 8 months after the study began. Due to the multifaceted nature of the intervention, however, it was difficult to determine which component of the interventions was beneficial.

In an example of a one-to-one support intervention, Harris et al. ${ }^{35}$ randomly placed women in inner London with chronic depression to volunteer befriending. A statistically significant effect on remission of symptoms was found after 1 year for the intervention group compared to wait-listed controls.

In some cases, investigators have sought to train individuals to elicit more frequent or higher-quality support from their existing networks. For example, Brand et al. ${ }^{36}$ recruited 51 adults with low perceived social support from organizations for singles and from divorce and bereavement support groups. Individuals were randomly assigned to a cognitive-behavioral intervention to address cognitive and behavioral barriers to forming rewarding interpersonal relationships. Participants in the intervention experienced significant increases in perceived family support, although not perceived support from friends. Symptoms of anxiety and depression were also unaffected by the intervention.

From the foregoing examples, it is evident that there are significant variations in the design, duration, timing, and types of social support intervention, such that few generalizable lessons can be gleaned from the existing literature. Some interventions have aimed to increase levels of perceived emotional support, others to augment informational support or instrumental support. Seldom have investigators sought to elucidate alternative mediating mechanisms by which the interventions were proposed to improve outcomes. We concur with Cohen et al. ${ }^{37}$ that more attention needs to be devoted to identifying the characteristics of individuals who benefit most (or least) from such interventions. The relevant issues here are to establish the feasibility of screening potential subjects for intervention, as well as to characterize the nature of the "dose-response" relationship between level and type of social ties and mental health. A low score on a social network screen does not necessarily reflect perceived need for greater support (and hence receptivity to support intervention). ${ }^{38}$ We also need to establish whether there is a threshold effect of 
social ties on mental health beyond which further increases in networks or support will have little effect, or whether there are benefits to be gained across a gradient of social connectedness. ${ }^{5}$ Finally, we need to consider interventions at the structural level (i.e., on social capital). As Gottlieb ${ }^{39}$ asserts:

\begin{abstract}
More thought needs to be given to ways of altering the structure and policies of community organizations and institutions, as well as work organizations in the private sector, to enhance the support their occupants receive. . . For example, system-level changes that make flexible work arrangements available to employees as a way of helping them achieve a healthier balance between their job and family responsibilities. ${ }^{\text {(p216) }}$
\end{abstract}

\title{
CONCLUSION
}

The association between social ties and mental health is securely established. Work remains to be carried out, however, in elucidating the mechanisms by which specific aspects of ties lead to the maintenance or improvement of psychological wellbeing - and hence, refining the approach to effective intervention. At the same time, research and interventions on social ties must take greater cognizance of the embeddedness of personal networks in the broader social structure.

\section{ACKNOWLEDGEMENT}

Dr. Kawachi is a core member of the MacArthur Foundation Network on SES and Health.

\section{REFERENCES}

1. Durkheim E. Suicide. New York: Free Press; 1951. (Originally published 1897.)

2. Barnett PA, Gotlib IH. Psychosocial functioning and depression: distinguishing among antecedents, concomitants, and consequences. Psychol Bull. 1988;104:97-126.

3. Cohen S, Wills TA. Stress, social support, and the buffering hypothesis. Psychol Bull. 1985;98:310-357.

4. Berkman LF, Glass T. Social integration, social networks, social support, and health. In: Berkman LF, Kawachi I, eds. Social Epidemiology. New York: Oxford University Press; 2000:137-173.

5. Cohen S, Underwood LG, Gottlieb BH. Social Support Measurement and Intervention. A Guide for Health and Social Scientists. New York: Oxford University Press; 2000.

6. Lin N, Ye X, Ensel WM. Social support and depressed mood: a structural analysis. $J$ Health Soc Behav. 1999;40:344-359.

7. Thoits PA. Social support as coping assistance. J Consult Clin Psychol. 1986;54:416423.

8. Kamarck, T., Manuck S, Jennings J. Social support reduces cardiovascular reactivity to psychological challenge: a laboratory model. Psychosom Med. 1990;52:42-58.

9. Kaslow NJ, Deering CG, Racusin GR. Depressed children and their families. Clin Psychol Rev. 1994;14:39-59.

10. Bowlby, J. Attachment and Loss. London, England: Hogarth Press; 1973.

11. Gunnar MR, Nelson CA. Event-related potentials in year-old infants: relations with emotionality and cortisol. Child Dev. 1994;65:80-94.

12. Oxman TE, Berkman LF, Kasl S, Freeman DH Jr, Barrett J. Social support and depressive symptoms in the elderly. Am J Epidemiol. 1992;135:356-368. 
13. Lee GR. Kinship and social support of the elderly: the case of the United States. Aging Soc. 1985;5:19-38.

14. Rook KS. The negative side of social interaction. J Pers Soc Psychol. 1984;46:10971108.

15. Fabrigoule C, Letenneur L, Dartigues J, Zarrouk M, Commenges D, Barberger-Gateau P. Social and leisure activities and risk of dementia: a prospective longitudinal study. $J$ Am Geriatr Soc. 1995;43:485-490.

16. Bassuk S, Glass T, Berkman L. Social disengagement and incident cognitive decline in community-dwelling elderly persons. Ann Intern Med. 1999;131:165-173.

17. Fratiglioni L, Wang H, Ericsson K, Maytan M, Winblad B. The influence of social network on the occurrence of dementia: a community-based longitudinal study. Lancet. 2000;355:1315.

18. Wethington E, McLeod JD, Kessler RC. The importance of life events for explaining sex differences in psychological distress. In: Barnett RC, Biener L, Baruch GK, eds. Gender and Stress. New York: The Free Press; 1987:144-156.

19. Belle D. Gender differences in the social moderators of stress. In: Barnett RC, Biener L, Baruch GK, eds. Gender and Stress. New York: The Free Press; 1987:257-277.

20. Belle D. Lives in Stress: Women and Depression. Beverly Hills, CA: Sage; 1982.

21. Stroebe MS, Stroebe W. Who suffers more? Sex differences in health risks of the widowed. Psychol Bull. 1983;93:279-301.

22. Lowenthal MJ, Haven C. Interaction and adaptation: intimacy as a critical variable. Am Sociol Rev. 1968;33:20-30.

23. Belle D. The impact of poverty on social networks and supports. Marriage Fam Rev. 1983;5:89-103.

24. Dressler WW, Badger LW. Epidemiology of depressive symptoms in black communities. J Nerv Ment Dis. 1985;173:212-220.

25. Corin E. The cultural frame: context and meaning in the construction of health. In: Amick BC III, Levine S, Tarlov AR, Chapman Walsh D, eds. Society and Health. New York: Oxford University Press; 1985:272-304.

26. Boissevain J. Friends of Friends. New York: St. Martin's Press; 1974.

27. Berkman LF. The role of social relations in health promotion. Psychosom Med. 1995; 57:245-254.

28. Faris REL, Dunham HW. Mental Disorders in Urban Areas. New York: Hafner; 1939.

29. Kawachi I, Kennedy BP, Lochner K, Prothrow-Stith D. Social capital, income inequality, and mortality. Am J Public Health. 1997;87:1491-1498.

30. Kawachi I, Berkman LF. Social cohesion, social capital, and health. In: Berkman LF, Kawachi I, eds. Social Epidemiology. New York: Oxford University Press; 2000:174190.

31. Brown GW, Harris TO. Social Origins of Depression: a Study of Psychiatric Disorder in Women. London: Tavistock; 1978.

32. Vachon M, Lyall W, Rogers J. A controlled study of self-help intervention for widows. Am J Psychiatry. 1980;137:1380-1384.

33. Mittleman MS, Ferris SH, Shulman E, et al. A comprehensive support program: effect on depression in spouse-caregivers of AD patients. Gerontologist. 1995;35:792-802.

34. Heller K, Thompson MG, Trueba PE, Hogg JR, Vlachos-Weber I. Peer support telephone dyads for elderly women: was this the wrong intervention? Am J Community Psychol. 1991;93:53-74.

35. Harris T, Brown GW, Robinson R. Befriending as an intervention for chronic depression among women in an inner city. I. Randomized controlled trial. $\mathrm{Br} J$ Psychiatry. 1999;174:219-224.

36. Brand EF, Lakey B, Berman S. A preventive, psychoeducational approach to increase perceived social support. Am J Community Psychol. 1995;23:117-135.

37. Cohen S, Gottlieb BH, Underwood LG. Social relationships and health. In: Cohen S, 
Underwood LG, Gottlieb BH, eds. Social Support Measurement and Intervention. A Guide for Health and Social Scientists. New York: Oxford University Press; 2000:3-25.

38. Rook KS, Underwood LG. Social support measurement and interventions. Comments and future directions. In: Cohen S, Underwood LG, Gottlieb BH, eds. Social Support Measurement and Intervention. A Guide for Health and Social Scientists. New York: Oxford University Press; 2000:311-334.

39. Gottlieb BH. Selecting and planning support interventions. In: Cohen S, Underwood LG, Gottlieb BH, eds. Social Support Measurement and Intervention. A Guide for Health and Social Scientists. New York: Oxford University Press; 2000:195-220. 